\title{
Physical Vapor Deposition of Multiphase Materials with Phase Nucleation via a Coupled Phase-Field Approach
}

\author{
James A. Stewart ${ }^{a, *}$ and Douglas E. Spearot ${ }^{b}$ \\ ${ }^{a}$ Institute for Nanoscience \& Engineering, University of Arkansas, Fayetteville, AR 72701 USA \\ ${ }^{\mathrm{b}}$ Department of Mechanical \& Aerospace Engineering, University of Florida, Gainesville, FL 32611 USA
}

\begin{abstract}
A coupled phase-field model is presented for simulating physical vapor deposition (PVD) of multi-phase materials, including the effects of phase nucleation. This model is utilized to study the role of initial substrate phase and temperature distributions, which are important experimental deposition parameters, on PVD of a generic allotropic metal with two stable phases. PVD simulations are performed for the deposition of a high temperature phase below its phase transition temperature, and for bicrystal and amorphous substrates with a Gaussian temperature distribution. Several general observations are made from these simulations. During the initial stages of PVD, the substrate phase distribution acts as a template for the growing phases. As the thin film continues to grow, the release of latent heat due to deposition creates a temperature gradient within the film, i.e., regions near the film surface become hotter than near the substrate. Additionally, a substrate with a defined temperature distribution that encompasses temperatures above and below the phase transition temperature, allows for distinguishable regions within the thin film where different phases are stable and continue to grow. Three-dimensional simulations provide additional insight into the role of substrate temperature and phase distribution on the resulting microstructure with different flux rates.
\end{abstract}

Keywords: Phase-Field Modeling, Physical Vapor Deposition, Microstructure Evolution, Solid-State Phase Transformation, Phase Nucleation

*Corresponding Author.

Email Address: jstewa@sandia.gov (J.A. Stewart)

Current Affiliation: Sandia National Laboratories, Albuquerque, NM 87185

(C) 2017. This manuscript version is made available under the Elsevier user license http://www.elsevier.com/open-access/userlicense/1.0/ 


\section{Introduction}

Considerable research has been devoted to thin films due to their application in optoelectronic and microelectronic devices, nanoelectromechanical systems, and protective coatings, among others. The microstructure of a thin film may be amorphous or consist of nanometer to micronsized regions that differ in phase (i.e., crystal structure), orientation, and chemical composition. The exact details of the surface morphology and subsurface structure generally influence its mechanical, electrical, and chemical properties.

Thin films are typically grown using vapor deposition techniques where the surface morphology and underlying microstructure (e.g., phase distribution, grain size, etc.) are a function of the deposition conditions and materials used [1-9]. During vapor deposition in some materials, multiple stable or metastable phases may nucleate and coarsen that will enhance or diminish the desired thin film properties (cf. [10-14]). As such, to elucidate links between vapor deposition processing conditions and thin film phase distribution or evolution, a predictive model must specifically consider solid state-phase transformations and phase nucleation in addition to critical aspects of thin film growth for the chosen deposition technique [9,15-17].

The objective of this work is to present a "first treatment" phase-field model to simulate simultaneous thin film growth and subsurface phase-evolution in a multiphase thin film with isotropic growth kinetics. Physical vapor deposition (PVD) is the growth process considered in this work because the composition of the depositing target material is conserved during transport to the substrate, and physical processes dominate over chemical reactions during vapor transport and thin film growth [2,3]. Therefore, chemical reactions and composition changes can be 
neglected in a phase-field model for multiphase PVD (provided the thin film is composed of a single element or a compound where all of the phases have the same stoichiometry, e.g., $\mathrm{Al}_{2} \mathrm{O}_{3}$ ). Within the PVD process, the critical aspects of thin film growth to be considered are surface tension, diffusion, non-local shadowing effects, and re-emission [1-3,18]. To the authors' knowledge, there has been no attempt in the literature to address concurrently thin film growth, subsurface phase evolution, and phase nucleation during PVD of multiphase materials.

Recently, Stewart and Spearot [19,20] developed two phase-field models for simulating PVD of a single-phase polycrystalline material. In that work, a traditional single free energy functional approach for describing the energetics of a PVD thin film system was compared to a coupled approach that treated the PVD growth process as being separate from the subsurface grain evolution process (i.e., different free energy expressions for each process). It was shown that the coupled phase-field approach is sufficient for determining qualitative descriptions of microstructure evolution during PVD (e.g., the influence of surface features on GB migration) which can act as a guide for developing more complex models, while the single free energy approach is necessary for a physically consistent description of these inherently connected PVD process mechanisms. In addition to prior work by the current authors, this work leverages previous modeling efforts on PVD growth dynamics [18], evolution in multiphase materials [21], and phase nucleation $[15,16]$, to incorporate the relevant aspects of the PVD growth process and subsurface phase evolution into a coupled phase-field model.

The proposed phase-field model is applied to simulate PVD of a generic allotropic metal with two stable phases and isotropic growth kinetics. These simulations allow for a study of initial 
substrate phase distribution and substrate temperature on the resulting phase distribution within the thin film. The deposition conditions simulated in this work are chosen to highlight the utility of the coupled PVD phase-field model in capturing general features of thin film growth with subsurface nucleation and evolution of multiple phases.

\section{Simulation Methodology}

In this section, the major components for modeling PVD, multiphase evolution, and classical phase nucleation within a coupled phase-field simulation framework are presented; full analyses of the motivating literature can be found in $[15,16,18,21]$. The formalism developed in this work to sequentially couple PVD thin film growth and subsurface phase evolution for modeling both processes is also presented.

\subsection{Physical Vapor Deposition}

To capture arbitrary surface morphology formation, surface tension and diffusion, and nonlocal shadowing effects during PVD, this work utilizes the interfacial growth model of Keblinski et al. [18], which was also used in [19,20] for capturing PVD processes of a single-phase material. To model vapor deposition, two field variables are introduced: $f(\boldsymbol{r}, t)$ and $g(\boldsymbol{r}, t)$. The first variable, $f(\boldsymbol{r}, t)$, describes the growing thin film where $f(\boldsymbol{r}, t) \approx 1$ defines a solid region, $f(\boldsymbol{r}, t) \approx-1$ defines a vapor region, and $f(\boldsymbol{r}, t) \approx 0$ defines interfaces. The second variable, $g(\boldsymbol{r}, t)$, describes the incident vapor density where $\mathrm{g}(\boldsymbol{r}, t) \approx 0$ defines a region of no incident flux and $\mathrm{g}(\boldsymbol{r}, t)>0$ defines the local density of incident vapor being transported to the thin film surface. The time evolution of these field variables is given in the following coupled non-dimensional equations of motion, 


$$
\begin{gathered}
\frac{\partial f}{\partial t}=\nabla^{2}\left(-f+f^{3}-2 a\left(\nabla^{2} f\right)\right)+B(\boldsymbol{\nabla} f)^{2} g+C \sqrt{(\nabla f)^{2} \mathrm{~g} \eta} \\
\frac{\partial \mathrm{g}}{\partial t}=\boldsymbol{\nabla}[D \nabla g-\boldsymbol{A g}]-B(\boldsymbol{\nabla} f)^{2} \mathrm{~g}
\end{gathered}
$$

Eq. (1) describes the growth and evolution of the solid, $f(\boldsymbol{r}, t)$, at the expense of the vapor, $\mathrm{g}(\boldsymbol{r}, t)$, while Eq. (2) describes the evolution of the incident vapor, $g(\boldsymbol{r}, t)$. The first term in Eq. (1) is formally the Cahn-Hilliard dynamics where the variational derivative of the double well free energy functional for this model has been calculated, providing an energy barrier between the vacuum and solid phases. This term allows the formation of arbitrary surface morphologies and accounts for both surface and bulk diffusion during thin film growth, where $a$ is the surface diffusion coefficient. The second term, coupling Eqs. (1) and (2), serves as the source term that leads to growth of the thin film at the expense of incident vapor, where $B$ controls the rate of vapor-to-solid aggregation. The last term provides thermal fluctuations following an uncorrelated Gaussian distribution, $\eta(\boldsymbol{r}, t)$, where the parameter, $C$, controls the overall strength of the noise. In Eq. (2), the first term is the diffusion equation modified for the presence of an external force, $\boldsymbol{A}$, where $D$ is the diffusion coefficient and $\boldsymbol{A}$ is the strength and direction to the incident vapor flux. The second term, opposite the second term in Eq. (1), is the sink that removes vapor in regions that have been converted to solid. Finally, an additional parameter, $b$, not explicitly included in Eqs. (1) and (2) is used to prevent solid growth in regions away from the interface in the vacuum region (i.e., $f(\boldsymbol{r}, t)<b$ ) and is constrained to the interval $-1<b<0$. 


\subsection{Multiphase Evolution}

The description of multiple phases within the thin film is motivated by the phase-field model for

multiphase systems developed by Steinbach et al. [21], which can be used to quantitatively model solid-solid and solid-liquid transformations. In later work, Steinbach et al. [22] extended this model to account more accurately for the conservation of interfacial stress. In this multiphase model, each of the $N$ phases are assigned a unique field variable, $p_{i}(\boldsymbol{r}, t)$, that varies between 0 and 1 . These variables correspond to the volume fraction of each phase at a given location, thus allowing for different phases to be modeled and distinguished. Therefore, at any given location in a $N$ phase system, the following constraint must be satisfied,

$$
\sum_{i=1}^{N} p_{i}(\boldsymbol{r}, t)=1
$$

A free energy functional for an $N$ phase system is constructed by considering all pairwise interactions, thus providing kinetic and potential energy terms that are dependent on the local $p_{i}(\boldsymbol{r}, t)$ values, their gradients, and temperature. These kinetic and potential energy terms capture bulk and interfacial energies and their differences for phases present in the local volume; defining the energy barriers for phase transformations. Since phase transformations are nonconserved phenomena, the equations of motion utilizing a free energy functional of this type are determined by Allen-Cahn dynamics, giving the following set of equations,

$$
\frac{\partial p_{i}}{\partial t}=\sum_{k \neq i}^{N} \frac{1}{\tau_{i k}}\left[\varepsilon_{i k}^{2}\left(p_{k} \nabla^{2} p_{i}-p_{i} \nabla^{2} p_{k}\right)-\frac{p_{i} p_{k}}{2 a_{i k}}\left(p_{k}-p_{i}-2 m_{i k}\right)\right]
$$


The parameters in Eq. (4) are as follows: $\tau_{i k}$ is a kinetic coefficient, $\varepsilon_{i k}^{2}$ defines the numerical interface thickness, $a_{i k}$ is a positive constant, and $m_{i k}$ is the coefficient for deviation from thermodynamic equilibrium that provides the local driving force as a function of temperature. These numerical parameters are related to the following physical thermodynamic quantities,

$$
\tau_{i k}=\frac{L_{i k} \lambda_{i k}}{T_{i k} \mu_{i k}} \quad \varepsilon_{i k}^{2}=\lambda_{i k} \sigma_{i k} \quad a_{i k}=\frac{\lambda_{i k}}{72 \sigma_{i k}} \quad m_{i k}=\frac{6 a_{i k} L_{i k}\left(T_{i k}-T\right)}{T_{i k}}
$$

where $L_{i k}$ is the latent heat released or consumed during the $i-k$ phase transformation, $\lambda_{i k}$ is the $i$ $k$ interface thickness, $T_{i k}$ is the temperature at which the $i-k$ phase transformation takes place, $\mu_{i k}$ is the $i-k$ interface mobility, and $\sigma_{i k}$ is the $i-k$ interfacial energy. All of these parameters depend on the phases that comprise the $i-k$ interface. As such, thermodynamic information for every material, phase, and interface is required to develop a quantitative phase-field model.

During PVD, substrates may be heated to a desired temperature, which inherently plays a major role in the distribution, nucleation, and evolution of phases within the growing thin film [2,3,23]. Furthermore, latent heat is released or absorbed by the thin film during deposition and phase transformations that can contribute thermal gradients to the system [24]. As such, temperature evolution within the thin film is a necessary aspect to be considered in a model describing the PVD process. Accordingly, the multiphase evolution expressions in Eqs. (4) and (5) are coupled to a temperature field through the heat conduction equation with a heat source / sink [24], 


$$
\rho_{i} C_{P} \frac{\partial T}{\partial t}=k_{T} \nabla^{2} T+\sum_{i=1, k>i}^{N} L_{i k} \frac{\partial p_{i}}{\partial t}
$$

Here, for the $i^{\text {th }}$ phase, $\rho_{i}$ is the density, $C_{P}$ is the heat capacity, and $k_{T}$ is the thermal conductivity. From the heat source / sink term, the latent heat contributes to the temperature evolution only when the field variable, $p_{i}(\boldsymbol{r}, t)$, is changing in time (e.g., at an interface where a phase transformation is occurring); otherwise, Eq. (6) is the traditional heat conduction equation.

\subsection{Phase Nucleation}

Thermally activated phase nucleation events during thin film growth are incorporated utilizing the approach of Simmons et al. $[15,16]$, which explicitly introduces nucleation sites into the phase-field model via classical nucleation theory and Poisson seeding. In this method, the classical nucleation rate, $J$, is given as $[15,16,25]$,

$$
J=\frac{3\left(\Delta G^{n u c}\right)^{2}}{4 \sqrt{\pi k_{B} T}\left(A_{S} \sigma_{i k}\right)^{3 / 2}} n \beta e^{\left(-\frac{\Delta G^{*}}{k_{B} T}\right)}
$$

In Eq. (7), the prefactor is the Zeldovich factor that accounts for the probability of nuclei to grow or dissolve, where $\Delta G^{n u c}$ is the difference in free energy required to form a nucleus of arbitrary size, $k_{B}$ is Boltzmann's constant, $T$ is temperature, $A_{S}$ is a geometric factor accounting for the shape of the nucleus, and $\sigma_{i k}$ is the interfacial energy between the $i$ - $k$ phases. The remaining parameters are as follows: $n$ is the number of atoms in the local phase-field volume, $\beta$ is the rate of critical nuclei growth (the rate at which individual atoms attach to a nuclei), and $\Delta G^{*}$ is the energy barrier required to form a stable nucleus of critical size, which can be calculated in two- 
dimensions as: $\Delta G^{*}=\pi \sigma_{i k}^{2} / \Delta G_{V}$, where $\Delta G_{V}$ is the change in free energy per area of a phasefield cell $[15,16,26]$.

Nuclei are randomly introduced into the system via the following algorithm [16,27]: (i) calculate the nucleation probability, $P=1-\exp (-J \Delta \mathrm{t})$, for each phase using the nucleation rate in Eq. (7) and the simulation time step, $\Delta \mathrm{t}$, (ii) generate a uniform random number, $R$, between 0 and 1 , (iii) add a nucleus to the chosen location when $P>R$. In a $N$ phase system, if more than one phase has a probability of nucleating at a given location, all of the nucleating phases are introduced into the local volume in equal fractions. Once the nuclei are introduced, evolution of these nuclei is governed by the corresponding phase-field equations (e.g., Eq. (4)).

\subsection{Coupling Physical Vapor Deposition and Multiphase Evolution}

The models outlined in the previous sections are coupled to create a sequential phase-field simulation approach. Within the same time step, PVD of the thin film occurs first followed by evolution of the underlying phases including nucleation events within the microstructure. Here, all PVD growth processes are described by the model in Section 2.1, thus determining the allowable growth and evolution of the thin film at a given location. The multiphase evolution in Section 2.2 is used to describe phase evolution beneath the thin film surface within the solid microstructure defined by the PVD model. Phase nucleation events occur once evolution of all phases has taken place, where nucleated phases begin evolving on the next time step. Therefore, the solid growth determined by the PVD model constrains evolution of the phases to occur only within those regions defined to be solid. With this coupled approach, features developed in the 
PVD model may influence phase evolution, but the presence of differing phases at the surface and interfaces does not influences PVD growth dynamics.

To apply this coupling condition, the following simple method is developed. The local solid density, $f_{d}(\boldsymbol{r}, t)$, in the PVD model is defined as $f_{d}(\boldsymbol{r}, t)=(f(\boldsymbol{r}, t)+1) / 2$, which has values between 0 and 1 [18]. This solid density describes the fraction of the local phase-field volume that is solid, where the difference $1-f_{d}(\boldsymbol{r}, t)$ is considered to be the fraction of the vapor phase. With this, the local solid density has a similar interpretation as the $p_{i}(\boldsymbol{r}, t)$ field variables in the multiphase model, which also define the volume fraction of each phase at a given location [19]. Therefore, to couple the PVD and multiphase evolution models in Sections 2.1 and 2.2, respectively, the condition that the sum of all field variables, $p_{i}(\boldsymbol{r}, t)$, describing only solid phases must equal the local solid density, $f_{d}(\boldsymbol{r}, t)$, at every time step, i.e.,

$$
\sum_{i=2}^{N} p_{i}(\boldsymbol{r}, t)=f_{d}(\boldsymbol{r}, t)
$$

Here, $p_{1}(\boldsymbol{r}, t)$ is assumed to describe the vapor phase and all $p_{i}$ with $i>1$ describe the solid phases within the thin film. Finally, $p_{1}+p_{i}$ (for $i=2, N$ ), must continue to equal 1 as defined by the original constraint in Eq. (3) for all multiphase field variables, $p_{i}(\boldsymbol{r}, t)$.

\subsection{Numerical Solution}

Equations (1), (2), and (4) are discretized and solved on a uniform 100 x 350 mesh using explicit first order and second order finite difference approximations for time and space, respectively. 
For these equations, periodic boundary conditions are applied in the directions parallel to the substrate ( $x$-axis) while anti-periodic boundary conditions are applied perpendicular to the substrate ( $y$-axis), i.e., the direction of thin film growth.

The initial configuration used in this work is that of a flat substrate located below $y=1$ and spans the entire $x$-axis where $f(\boldsymbol{r}, 0)=1$ and $\mathrm{g}(\boldsymbol{r}, 0)=0$. Below the $y=1$ boundary, these values are held fixed to provide a rigid substrate. The region above the substrate is taken to be the vacuum region with an incident vapor flux where $f(\boldsymbol{r}, 0)=-1$ and $\mathrm{g}(\boldsymbol{r}, 0)=\mathrm{g}_{0}$. Above the $y=350$ boundary, these values are held fixed to provide a continuous vapor source for deposition. Within the initial substrate region, a phase distribution is constructed and held fixed where $p_{1}=0$ and the values of $p_{i}$ (for $i=2, N$ ) depend on the volume fraction ascribed to each phase at each mesh point. Within the initial vacuum region, only vapor exists so $p_{1}=1$ and all other $p_{i}$ equal 0 . Finally, a temperature distribution is defined for the entire system within the substrate and vapor regions. Within the substrate and above the $y=350$ boundary, this temperature distribution is held fixed. Taking the temperature to be fixed at these boundaries and choosing the $y$-axis to be larger than the $x$-axis allows for heat released during deposition to diffuse into and away from the thin film and eventually be removed so that large temperature accumulations are prevented (i.e., the substrate and thin film are not thermally isolated from the PVD deposition chamber), which would occur in a closed system and influence the thermodynamics of phase evolution.

The choice of domain size is determined by performing a series of test simulations with sizes ranging between $100 \times 100$ and $100 \times 500$, focusing on the temperature distribution of the 
system and specifically the average temperature at $y=100$ during each simulation. These results are plotted in Figure 1. From these test simulations, it is determined that a domain size of $100 \times 350$ provides a reasonably converged temperature evolution, i.e., minor differences are observed in the thin film temperature evolution with domain sizes larger than $100 \times 350$, while still requiring a reasonable amount of computing time. Figure 1 shows that there is a distinct change in the rate of temperature increase during each simulation; this corresponds to the formation of columnar surface features and subsurface porosity, from an initially flat growing thin film, as will be shown in Section 3. Note, thin film features are fully formed within the first $100 y$-axis mesh points; therefore, simulation results in the following sections are presented on the corresponding $100 \times 100$ mesh.

\section{Simulation Results and Discussion}

The parameters used in this work for PVD, phase evolution, and phase nucleation are outlined in Section 3.1 for a pure allotropic metal with two possible solid phases. Section 3.2 presents simulation results for PVD of a high temperature phase at low deposition temperatures (e.g., below the phase transition temperature) with a single crystal substrate. Section 3.3 presents simulation results for PVD on amorphous and bicrystal substrates with a Gaussian temperature distribution that encompasses the phase transition temperature. Finally, Section 3.4 extends the PVD phase-field model to three dimensions to illustrate the resulting microstructure, phase distribution, and temperature profile with different vapor flux rates.

\subsection{Model Parameters}


The non-dimensional parameters in Eqs. (1) and (2) are chosen to produce columnar and porous features during purely vertical PVD simulations as described in $[18,19,20]$; these parameters are summarized in Table 1. The non-dimensional spatial discretization is taken to be 1 as in Keblinski et al. [18] while the non-dimensional temporal discretization is taken to be $10^{-5}$.

Table 1. Non-dimensional parameters used to simulate columnar and porous growth during vertical PVD.

\begin{tabular}{cccccccc}
\hline Parameter & $a$ & $b$ & $A_{y}$ & $B$ & $C$ & $D$ & $g_{0}$ \\
\hline Value & 0.5 & -0.8 & -0.6 & 10 & 2.5 & 0.01 & 1 \\
\hline
\end{tabular}

The physical quantities for phase evolution in Eqs. (5) and (6) need to be defined for each phase and combination of phases. The values chosen in this work are summarized in Table 2 and represent reasonable values for an allotropic metal with two stable phases, $\alpha$ and $\beta$, where $\alpha$ is taken to be the stable phase below the transformation temperature and $\beta$ is the stable phase at high temperatures. Ti is used as motivation for the choice of parameters in this work as physical data is readily available $[28,29]$ and experimental PVD observations have highlighted concurrent deposition and growth of $\alpha$-Ti and $\beta$-Ti as a function of temperature [23]. However, the current model is not limited to this material. For simplicity, the interface energies and mobilities are taken to be constant. Furthermore, $L_{k i}=-L_{i k}$ and $\mu_{k i}=-\mu_{i k}$ depending on the direction of the phase transformation [21]. The remaining quantities are taken to be constant for all phases and are given as: $\rho_{i}=4.5 \mathrm{~g} / \mathrm{cm}^{3}, C_{P}=0.5 \mathrm{~J} / \mathrm{g} \cdot \mathrm{K}$, and $k_{T}=0.3 \mathrm{~W} / \mathrm{cm} \cdot \mathrm{K}$. For both Eqs. (5) and (6), the spatial and temporal discretizations are chosen to be $10^{-5} \mathrm{~cm}$ and $10^{-11}$, respectively, which corresponds to a physical domain size of $10 \mu \mathrm{m} \times 10 \mu \mathrm{m}$. 
Table 2. Physical parameters used to simulate subsurface two-phase evolution during PVD [2831].

\begin{tabular}{lccccc}
\hline Parameter & $L_{i k}\left(\mathrm{~J} / \mathrm{cm}^{3}\right)$ & $\lambda_{i k}(\mathrm{~cm})$ & $T_{i k}(\mathrm{~K})$ & $\sigma_{i k}\left(\mathrm{~J} / \mathrm{cm}^{2}\right)$ & $\mu_{i k}(\mathrm{~cm} / \mathrm{s} \cdot \mathrm{K})$ \\
\hline$\alpha-\beta$ & $3.15 \cdot 10^{2}$ & $5 \cdot 10^{-5} \mathrm{~cm}$ & 1200 & $5.175 \cdot 10^{-6}$ & 0.035 \\
\hline$\alpha-$ vapor & $4 \cdot 10^{4}$ & $5 \cdot 10^{-5} \mathrm{~cm}$ & 3500 & $1.5 \cdot 10^{-5}$ & $10^{-6}$ \\
\hline$\beta$ - vapor & $4 \cdot 10^{4}$ & $5 \cdot 10^{-5} \mathrm{~cm}$ & 3500 & $1.95 \cdot 10^{-5}$ & $10^{-6}$
\end{tabular}

The nucleation rate in Eq. (7) has several quantities that need to be defined. Here, the expression is simplified to reduce the complexity and number of unique parameters. The parameters $\beta$ and $\left(\Delta G^{n u c}\right)^{2}$, both of which are temperature dependent $[16,26]$, and $A_{S}^{3 / 2}$ are combined into a single parameter, $\beta^{*}$. Thus, the nucleation rate used in this work has the following form,

$$
J=\frac{3}{4 \sqrt{\pi k_{B} T}\left(\sigma_{i k}\right)^{3 / 2}} n \beta^{*} e^{\left(-\frac{\Delta G^{*}}{k_{B} T}\right)}
$$

where $\beta^{*}$ becomes a temperature-dependent numerical frequency factor that controls the amplitude of the nucleation rate and the number of nuclei to be introduced. This parameter, taken to be $\beta^{*}=0.25 \cdot 10^{-35}$, is chosen by performing a series of test simulation using the parameters below to achieve reasonable production of multiple nuclei during the simulation. The number of atoms in each local phase-field volume, $n$, is assumed to be constant and taken as $2 \cdot 10^{5}$; this is an approximation based on the volume of a phase-field mesh point and the number of atoms per $\beta$-Ti unit cell. To determine the energy required to form a stable nucleus in $2 \mathrm{D}, \Delta G^{*}$, the free energies, $G_{V}^{i}$, for each phase are required. In this work, the free energies for the $\alpha$ and $\beta$ phases are approximated as linear functions of temperature and are given as: $G_{V}^{\alpha}=0.025 T+$ 
$14.0 \mathrm{~J} / \mathrm{mol} \cdot \mathrm{K}$ and $G_{V}^{\beta}=0.015 T+26.0 \mathrm{~J} / \mathrm{mol} \cdot \mathrm{K}$, where these functions intersect at $T=$ $1200 K$ (i.e., the solid state phase transformation temperature). These functions are motivated by the temperature dependence of the free energies of Ti [29]; however, the free energy values and slopes of these relationships are modified to amplify the difference in free energies as the temperature deviates from the transition temperature. Below the transformation temperature, the free energy for $\alpha$ is lower than for $\beta$, thus providing a higher probability of $\alpha$ nuclei production within the $\beta$ phase while a smaller but non-zero nucleation probability is calculated for $\beta$ production within the $\alpha$ phase. Opposite arguments can be made for free energies and nucleation probabilities above the transformation temperature.

\subsection{Low Temperature PVD of a High Temperature Phase}

PVD simulations are performed on a purely $\beta$-phase substrate at temperatures below the $\alpha-\beta$ transition temperature to model low temperature deposition of a high temperature phase. Recall, the phase transition temperature is defined as $T=1200 \mathrm{~K}$, while these simulations are performed at $T=900 \mathrm{~K}$, as shown in Figure 2, and $T=1080 \mathrm{~K}$, as shown in Figure 3. Experimentally, low temperature PVD simulations of a high temperature phase is motivated by prior studies that explored the feasibility of depositing $\alpha-\mathrm{Al}_{2} \mathrm{O}_{3}$ at low substrate temperatures, to take advantage of its high hardness, and the role of the substrate temperature on microstructure development $[32,33]$.

In both figures, the thin film initially grows with the $\beta$-phase, followed by the formation of several $\alpha$-phase nuclei, which grow in size as it is the stable phase below the $\alpha-\beta$ transition temperature. As thin film growth continues, the temperature difference between the thin film 
surface and the substrate increases due to latent heat release during deposition. Thus, the $\alpha-\beta$ transition temperature is crossed during deposition, which occurs earlier in Figure 3 than in Figure 2 due to the higher initial deposition temperature. Within the region of the film near the transition temperature, the nucleation probability rapidly increases as the $\alpha$ - and $\beta$-phases have free energies that become nearly identical and a very large number of $\alpha$-phase nuclei are formed. The rapid formation and growth of multiple stable $\alpha$-phase nuclei lead to a horizontal band of stable $\alpha$-phase within the thin film corresponding to the region where the $\alpha-\beta$ transition temperature is located within the solid. For both simulations, within the solid region above the $\alpha$ $\beta$ transition temperature, the stable phase is the $\beta$-phase and continues to grow with no regions of $\alpha$-phase nucleation. However, in the solid region below the $\alpha-\beta$ transition temperature, the preexisting $\beta$-phase is eventually consumed by the formation and growth of $\alpha$-phase nuclei, which is the stable phase in this temperature regime.

Furthermore, as the thin film grows, surface features and subsurface porosity are predicted by the solution of Eqs. (1) and (2) for PVD. This roughness transition can be identified for all simulations in this work in Figures 2-5(e), which track the global volume fraction evolution of each phase within the domain, as a change in the rate of consumption of the vapor phase (indicated by the inset arrows). This increase in the rate of vapor phase consumption is due to the increase in surface area of the thin film due to the roughening transition. Note, vapor consumption rate changes also correspond to the change in the rate of average temperature increase as shown in Figure 1.

\subsection{PVD on Substrates with a Temperature Distribution}


PVD simulations are performed on amorphous and bicrystal substrates, shown in Figures 4 and 5, using a temperature distribution that follows a Gaussian profile where the center of the system is taken to be $T=1350 \mathrm{~K}$ and decreases horizontally outward to $T=1000 \mathrm{~K}$ at the domain boundaries. These PVD simulations are experimentally motivated by the work of Bunshah and Juntz [23], who showed that regions of stable $\alpha$-Ti or $\beta$-Ti formation were influenced by the presence of temperature gradients within the substrate

In Figure 4, the amorphous substrate phase distribution initially acts as a template for the phase distribution within the growing thin film. However, due to the size and shape (i.e., high density of interfaces) of the $\alpha$-and $\beta$-phase regions throughout the thin film, the stable phases associated with each temperature regime rapidly begin to coarsen. As thin film growth continues, the latent heat released during the deposition process increases the temperature near the thin film surface to be sufficiently large so that phase nucleation is reduced and the only stable phase in this region is the $\beta$-phase, which subsequently becomes the only growing phase. As such, the remaining $\alpha$ phase regions exist at low temperature regions deep within the thin film near the substrate where the Gaussian temperature distribution is held fixed. Note, if the simulation were stopped between Figures 4(a) and 4(b), corresponding to very thin film growth, a microstructure resembling the substrate phase distribution would be possible, in agreement with experiments [9]. Finally, due to the high density of interfaces of the $\alpha$ - and $\beta$-phase regions, nucleation of phases has a negligible influence on microstructure evolution and any nuclei that form rapidly dissolve.

In Figure 5, the bicrystal substrate again acts as a template for the initial phase distribution within the growing thin film. Minimal phase nucleation occurs in temperature regions away from 
the $\alpha-\beta$ transition temperature where the nuclei either dissolve or are rapidly consumed by the coarsening of the existing $\beta$-phase. Deep within the thin film, near the substrate, where the $\alpha-\beta$ transition temperature is crossed, the $\alpha$-phase nuclei that form within the $\beta$-phase region (e.g., bottom right of Figure 5 (a)-(d)) grow and consume the $\beta$-phase while the $\beta$-phase nuclei that form within the $\alpha$-phase region (e.g., bottom left of Figure 5 (a)-(d)) rapidly dissolve to the $\alpha$ phase. Therefore, regions of stable $\alpha$-phase are able to exist within the $\beta$-phase dominant thin film due to the temperature distribution created from latent heat release and the fixed Gaussian temperature distribution of the substrate. As the thin film continues to grow, the temperature within the thin film near the surface increases causing the $\beta$-phase to be the stable phase, which is illustrated by the $\beta$-phase consuming the $\alpha$-phase region on both sides (due to the periodic boundary conditions). However, in this simulation, the consumption of the $\alpha$-phase region will require a longer time (compared to Fig. 4) due to its initial size and the lack of $\beta$-phase subregions or nuclei to coarsen and accelerate the process.

\subsection{Three-Dimensional Microstructure and Temperature Distributions}

The two-dimensional multiphase PVD model is extended to three dimensions and PVD simulations are performed with two different flux rates on a bicrystal substrate to illustrate that the conclusions drawn using a simplified two-dimensional model in the prior sections are valid in three-dimensions. The results of these PVD simulations are illustrated in Figures 6 and 7. The deposition temperature is taken to be $T=1350 \mathrm{~K}$, which is above the $\alpha-\beta$ transition temperature. Furthermore, to decrease computational demand, the simulation mesh is taken to be $80 \times 80 \times 80$ and the phase nucleation algorithm is turned off, as it made a minimal contribution in the prior 
simulations presented with regions of the film near this temperature regime. Periodic boundary conditions are applied to the added dimension (z-direction).

PVD simulation results for $A_{y}=-0.6$ and $A_{y}=-2.5$ are illustrated in Figures 6 and 7, respectively, where (a) illustrates the resulting microstructure, i.e., $f(\boldsymbol{r}, t)$ field, predicted by Eq. (1), (b) illustrates the resulting multiphase evolution within the thin film solid regions, and (c) illustrates the resulting temperature distribution. The cubic nature of the simulation domain and the fixed temperature boundary condition at the top of the simulation domain causes heat to be removed faster than the previous two-dimensional simulations. In Figure 6, the formation of columnar structures and subsurface porosity is observed. Additionally, any cross-section of these three-dimensional simulations would effectively correspond to a two-dimensional simulation at this uniform temperature. Within the solid regions, the initial bicrystal substrate promotes the growth of $\alpha$ - and $\beta$-phases. However, as the deposition temperature is above the $\alpha-\beta$ transition temperature, the $\alpha$-phase transitions to the $\beta$-phase throughout the simulation and begins near the substrate. Similarly, Figure 7 illustrates the formation of a less dense thin film with a diffuse subsurface microstructure where the columnar features are thinner, more abundant, and less distinct than in Figure 6. Furthermore, with a larger incident vapor flux rate than Figure 6, there is less time for the $\alpha$-phase to transition to the stable $\beta$-phase during the simulation, leading to slower consumption of the $\alpha$-phase. Finally, Figures 6(c) and 7(c) illustrate the formation of a temperature gradient within the solid due to latent heat release due to deposition where the thin film becomes hotter at the surface than near the substrate, which leads to different phase evolution kinetics in each region. 


\section{Summary and Conclusions}

A "first treatment" phase-field model is developed and applied that couples previous modeling efforts in a sequential approach to simulate thin film growth, subsurface phase evolution, and phase nucleation in multiphase thin films. To illustrate this model's capability in capturing growth and evolution aspects of the PVD process, simulations are performed considering a generic allotropic metal with two stable solid phases and isotropic growth kinetics. Specifically, this model is used to study the role of initial substrate phase and temperature distribution on the resulting thin film microstructure by performing simulations of deposition of a high temperature phase below the phase transition temperature, and simulations of temperature distribution effects on microstructure evolution using amorphous and bicrystal substrates.

It is important to note several limitations, which have been assumed in this first treatment for simplicity. First, real materials have grain orientations and grain boundaries associated with the phases present, which are neglected in this work but were the focus for PVD simulations of a single-phase material in Stewart and Spearot [19,20]. Furthermore, these grains may have preferred growth directions or anisotropies that influence PVD growth dynamics, which are also neglected. Second, different attachment kinetics between the vapor phase and the phases present at the thin film surface are neglected, which can also influence surface diffusion and growth dynamics $[7,34]$. Specific to this work, the features developed by the PVD model are able to influence the phase evolution of the underlying microstructure, but the underlying features are not able to influence surface growth dynamics. Finally, the PVD equations of motion and parameters were constructed to be non-dimensional whereas the multiphase and phase nucleation models can be linked to physical quantities making them quantitative. 
Regardless of the above limitations, the simulation results presented here allow several general observations to be made regarding the connection between substrate phase distribution, temperature, and the resulting thin film microstructure. First, the substrate phase distribution tends to act as a template to the depositing and growing phases within the thin film until the thin film becomes sufficiently thick for the thermodynamics to control evolution. As the thin film grows, latent heat is released at the thin film surface causing a temperature gradient to form where the regions near the thin film surface become hotter than near the substrate. This temperature gradient causes the nucleation rate of the phases to change throughout the thickness of the thin film, where the nucleation rate is decreased in higher temperature regions, thus causing nucleation to occur mostly in the regions away from the thin film surface or near the substrate (see Figs. 2 and 3). The value for the nucleation parameter, $\beta^{*}$, needs to be carefully chosen as a function of temperature so that neither excessive nucleation nor lack of nucleation occurs, depending on the material system. In future studies, the nucleation parameter, $\beta^{*}$, can be posed as a function of spatial position within the model so that heterogeneous nucleation at the film/substrate or film/vapor interfaces is predicted, as opposed to the homogeneous nucleation behavior explored in this study.

As observed in Figs. 4 and 5, imposing a substrate temperature distribution allows for regions within the thin film where different phases are stable and able to grow or nucleate. Therefore, the substrate phase distribution and temperature along with material specific quantities play a significant role in the growth and underlying evolution of multiphase thin films as captured by this model. This is most evident in Figs. 6 and 7, which illustrate the resulting microstructure, 
phase distribution, and thin film temperature profile as a function of incident vapor flux rate. Collectively, the general observations made here are consistent with experimental studies of vapor deposited metals and alloys that investigate and characterize the influence of deposition conditions on the formation of thin film microstructure [12,13,23]. Primarily, these studies reported that temperature distributions within the film or substrate and a varying vapor flux rate allow for the formation and growth of distinct regions containing different phases and grain distributions due to their local thermodynamic stability -- as predicted by the present model.

\section{Acknowledgments}

This research was supported by the National Science Foundation under grant CMMI \#0954505.

The simulations in this work were performed at the Arkansas High Performance Computing Center (AHPCC), supported in part by the National Science Foundation through grants MRI \#0722625, MRI-R2 \#0959124, ARI \#0963249, \#0918970, and a grant from the Arkansas Science and Technology Authority.

\section{References}

[1] Karabacak, T. (2011) Thin-film growth dynamics with shadowing and re-emission effects. Journal of Nanophotonics, 5, 1 - 18.

[2] Thornton, J.A. (1977) High Rate Thick Film Growth. Annual Reviews of Materials Science, 7, 239 - 260.

[3] Plummer, J.D., Deal, M. and Griffin, P.D. Silicon VLSI Technology: Fundamentals, Practice, and Modeling. Prentice Hall, 2000. Print.

[4] Boettinger, W.J., Warren, J.A., Beckermann, C. and Karma, A. (2002) Phase-Field Simulation of Solidification. Annual Review of Materials Research, 32, 163 - 194.

[5] Chen, L.Q. (2002) Phase-Field Models for Microstructure Evolution. Annual Review of Materials Research, 32, 113 - 140. 
[6] Nestler, B. Garcke, H. and Stinner, B. (2005) Multicomponent alloy solidification: Phasefield modeling and simulations. Physical Review E, 71, 1 - 6.

[7] Pusztai, T. Bortel, G. and Granasy, L. (2005) Phase field modeling of polycrystalline freezing. Materials Science and Engineering A, 413 - 414, 412 - 417.

[8] Wang, X. (2015) Modeling and understanding materials microstructure evolution driven by interface energy. Computational Materials Science, 107, 1 - 7.

[9] Petrov, I., Barna, P.B., Hultman, L. and Greene, J.E. (2003) Microstructural evolution during thin film growth. Journal of Vacuum Science and Technology A, 21, S117 - S128.

[10] Braic, M., Braic, V., Vladescu, A., Zoita, C.N. and Balaceanu, M. (2014) Solid solution or amorphous phase formation in TiZr-based ternary to quinternary multi-principleelement films. Progress in Natural Science: Materials International, 24, 305 - 312.

[11] Dragoo, A.L. and Diamond, J.J. (1967) Transitions in Vapor-Deposited Alumina from $300^{\circ}$ to $1200^{\circ}$. Journal of the American Ceramic Society, 50, 568 - 574.

[12] Kabla, M., Seiner, H., M. Musilova, M., Landa, M. and Shilo, D. (2014) The relationships between sputter deposition conditions, grain size, and phase transformation temperatures in NiTi thin films. Acta Materialia, 70, 79 - 91.

[13] Krzanowski, J.E. (2004) Phase formation and phase separation in multiphase thin film hard coatings. Surface \& Coatings Technology, 188 - 189, 376 - 383.

[14] Ruppi, S. (2005) Deposition, microstructure and properties of texture-controlled CVD aA12O3 coatings. International Journal of Refractory Metals \& Hard Materials, 23, 306 316.

[15] Simmons, J.P., Shen, C. and Wang, Y. (2000) Phase Field Modeling of Simultaneous Nucleation and Growth by Explicitly Incorporating Nucleation Events. Scripta Materialia, 43, 935 - 942.

[16] Simmons, J.P., Wen, Y., Shen, C. and Wang, Y.Z. (2004) Microstructural development involving nucleation and growth phenomena simulated with the Phase Field method. Materials Science and Engineering A, 365, 136 - 143.

[17] Yang, Y.G., Johnson, R.A. and Wadley, H.N.G. (1997) A Monte Carlo Simulation of the Physical Vapor Deposition of Nickel. Acta Materialia, 45, 1455 - 1468.

[18] Keblinski, P., Maritan, A., Toigo, F., Messier, R. and Banavar, R. (1996) Continuum model for the growth of interfaces. Physical Review E, 53, 759 - 778. 
[19] Stewart, J.A. and D.E. Spearot (2016) Phase-Field Models for Simulating Physical Vapor Deposition and Grain Evolution of Isotropic Single-Phase Polycrystalline Thin Films. Computational Materials Science, 123, 111 - 120.

[20] Stewart, J.A. and D.E. Spearot (2017) Phase-Field Simulations of Microstructure Evolution During Physical Vapor Deposition of Single-Phase Thin Films. Computational Materials Science, 131, 170 - 177.

[21] Steinbach, I., Pezzolla, F., Nestler, B., Seeßelberg, M., Prieler, R., Schmitz, G.J. and Rezende, J.L.L. (1996) A phase field concept for multiphase systems. Physica D, 94, 135 $-147$.

[22] Steinbach, I. and Pezzolla, F. (1999) A generalized field method for multiphase transformations using interface fields. Physica D, 134, 385 - 393.

[23] Bunshah, R.F. and Juntz, R.S. (1973) Influence of Condensation Temperature on Microstructure and Tensile Properties of Titanium Sheet Produced by High-Rate Physical Vapor Deposition Process. Metallurgical Transactions, 4, 21 - 26.

[24] Xu, H., Matkar, R. and Kyu, T. (2005) Phase-field modeling on morphological landscape of isotactic polystyrene single crystals. Physical Review E, 72, 011804 (9).

[25] Clouet, E. (2009) Modeling of Nucleation Processes. D. Furrer and S.L. Semiatin (Eds.), ASM Handbook Volume 22A: Fundamentals of Modeling for Metals Processing (pp. 203 - 219). ASM International.

[26] Oxtoby, D.W. (1998) Nucleation of First-Order Phase Transitions. Accounts of Chemical Research, 31, 91 - 97.

[27] Heo, T.W. and Chen, L.Q. (2014) Phase-Field Modeling of Nucleation in Solid-State Phase Transformations. JOM, 66, 1520 - 1528.

[28] Lide, D.R. (2002) CRC Handbook of Chemistry and Physics (83rd ed.). CRC Press: Boca Raton.

[29] Robie, R.A., Hemingway, B.S. and Fisher, J.R. (1984) Thermodynamic Properties of Minerals and Related Substances at $298.15 \mathrm{~K}$ and 1 Bar $\left(10^{5}\right.$ Pascals) Pressure and at Higher Temperatures. U.S. Geological Survey Bulletin 1452. United States Government Printing Office: Washington.

[30] Olmstead, D.L., Holm, E.A. and Foiles, S.M. (2009) Survey of computed grain boundary properties in face-centered cubic metals - II: Grain boundary mobility. Acta Materialia, 57, 3704 - 3717.

[31] Wang, S. (2006) Molecular dynamics simulation study of grain boundary migration in nanocrystalline Pd (Doctoral Dissertation). Louisiana State University. 
[32] Cloud, A.N., Aryasomayajula, A., Bhat, D.G., Gordon and M.H. (2008) Determining substrate temperature in an AC inverted cylindrical magnetron sputtering PVD system. Surface and Coatings Technology, 202, 1564 - 1567.

[33] Cloud, A.N., Canovic, S., Abu-Safe, H.H., Gordon, M.H. and Halvarsson, M. (2008) TEM investigation of alpha alumina films deposited at low temperature. Surface and Coatings Technology, 203, $808-811$.

[34] Attard, G. and Barnes, C. (1998) Surfaces. Oxford: Oxford University Press. 


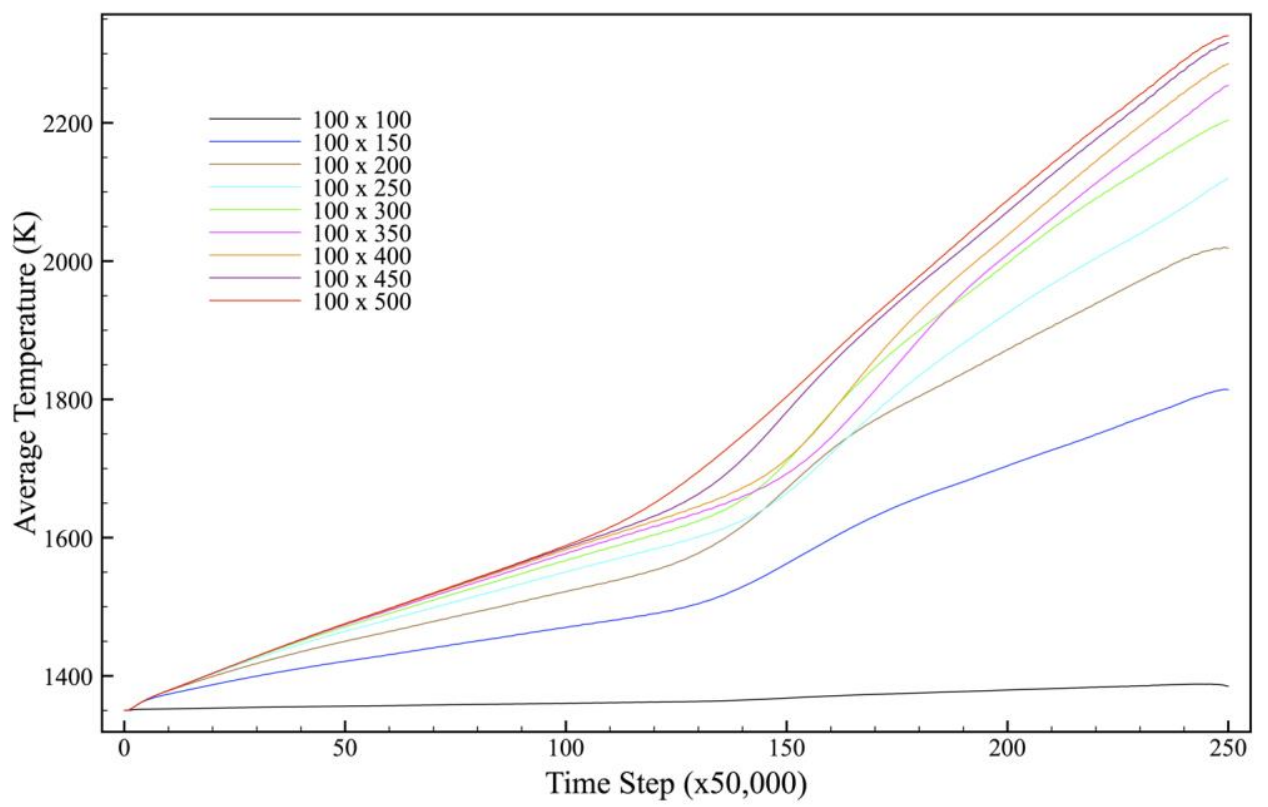

Figure 1. Test PVD simulations for varying domain sizes showing the average temperature evolution at $y=100$.
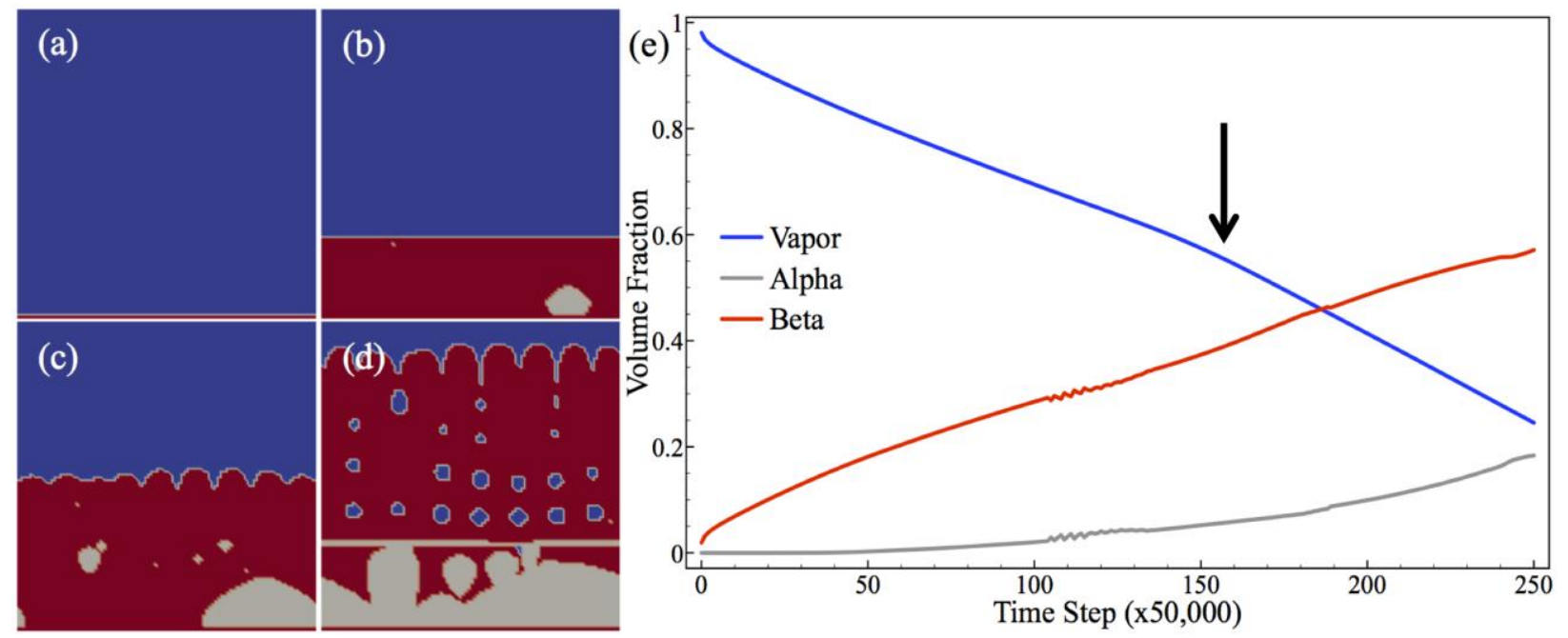

Figure 2. Simulated PVD on a $\beta$-phase substrate with $T=900 \mathrm{~K}$ at time steps (a) 0 , (b) 3750000 , (c) 7500000, and (d) 12500000. The phase with the maximum local volume fraction is plotted. (e) Global volume fraction evolution of each phase during the PVD simulation. The constant volume fraction of the substrate is included in this calculation to provide a consistent description with the images on the left. 

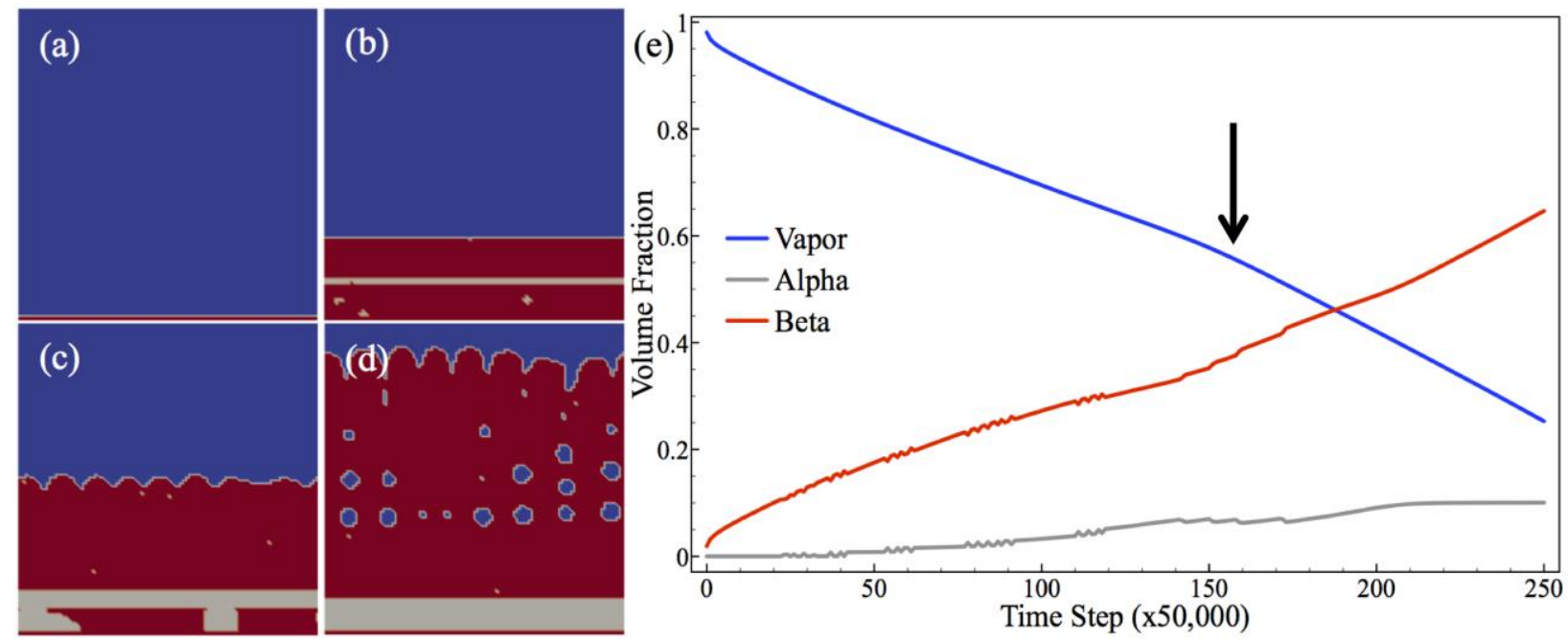

Figure 3. Simulated PVD on a $\beta$-phase substrate with $T=1080 \mathrm{~K}$ at time steps (a) 0 , (b) 3750000 , (c) 7500000, and (d) 12500000. The phase with the maximum local volume fraction is plotted. (e) Global volume fraction evolution of each phase during the PVD simulation. The constant volume fraction of the substrate is included in this calculation to provide a consistent description with the images on the left.
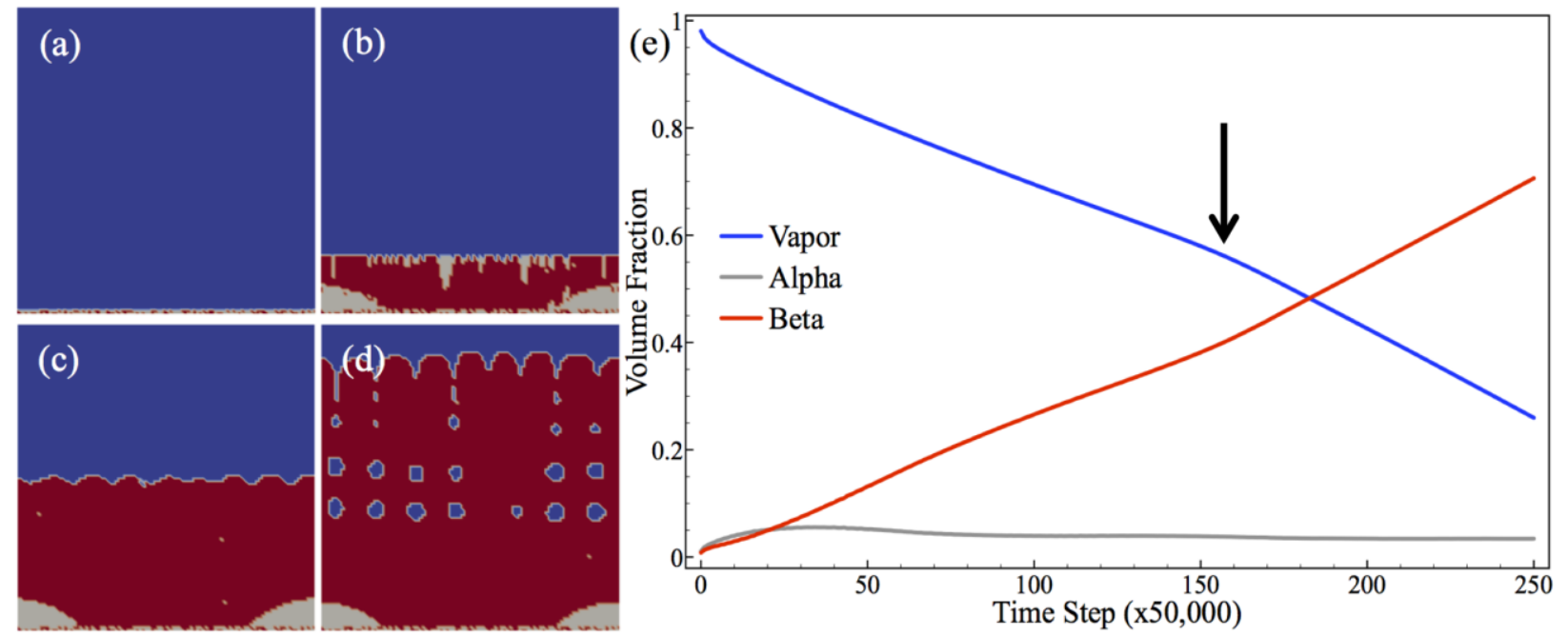

Figure 4. Simulated PVD on an amorphous substrate with a temperature distribution that follows a Gaussian profile with $T=1350 \mathrm{~K}$ in the middle of the system and $T=1000 \mathrm{~K}$ at the horizontal boundaries at time steps (a) 0, (b) 2500000, (c) 7500000, and (d) 12500000. The phase with the maximum local volume fraction is plotted. (e) Global volume fraction evolution of each phase during the PVD simulation. The constant volume fraction of the substrate is included in this calculation to provide a consistent description with the images on the left. 

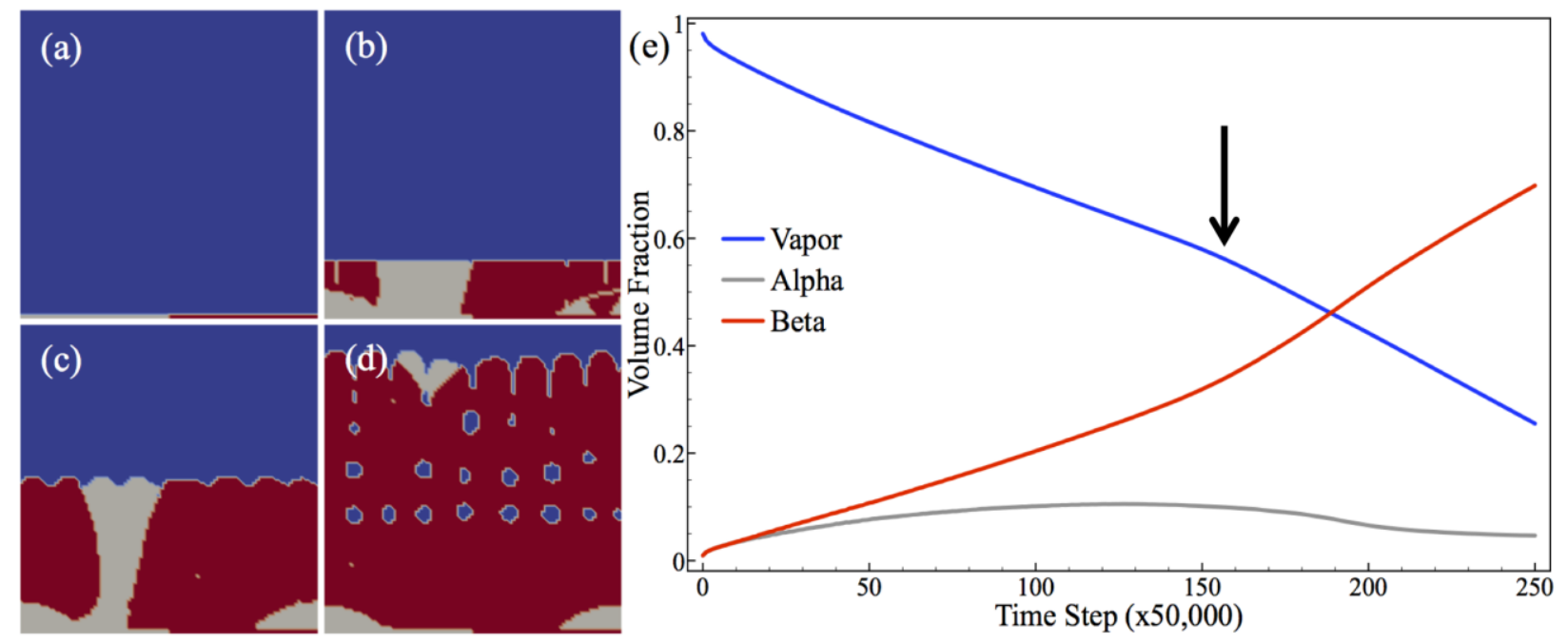

Figure 5. Simulated PVD on a bicrystal substrate with a temperature distribution that follows a Gaussian profile with $T=1350 \mathrm{~K}$ at the middle of the system and $T=1000 \mathrm{~K}$ at the horizontal boundaries at time steps (a) 0, (b) 2500000, (c) 7500000, and (d) 12500000. The phase with the maximum local volume fraction is plotted. (e) Global volume fraction evolution of each phase during the PVD simulation. The constant volume fraction of the substrate is included in this calculation to provide a consistent description with the images on the left.

(a)

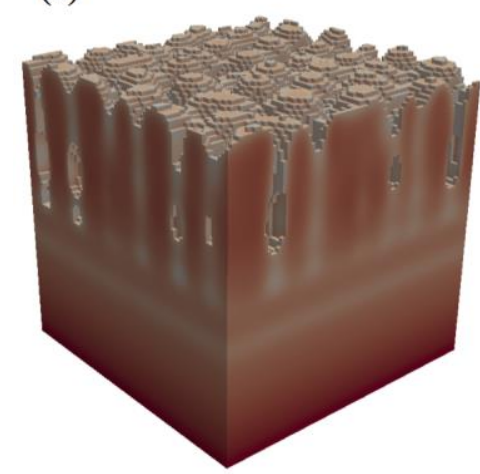

(b)

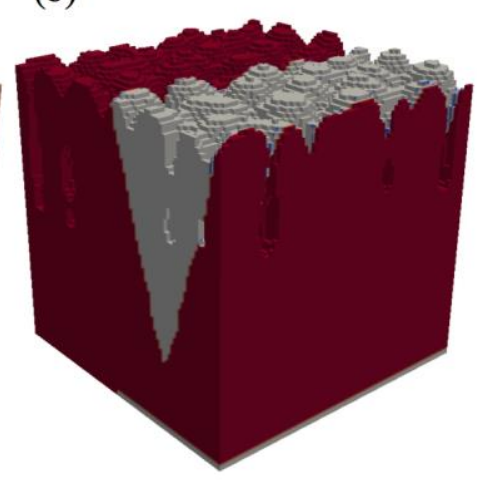

(c)

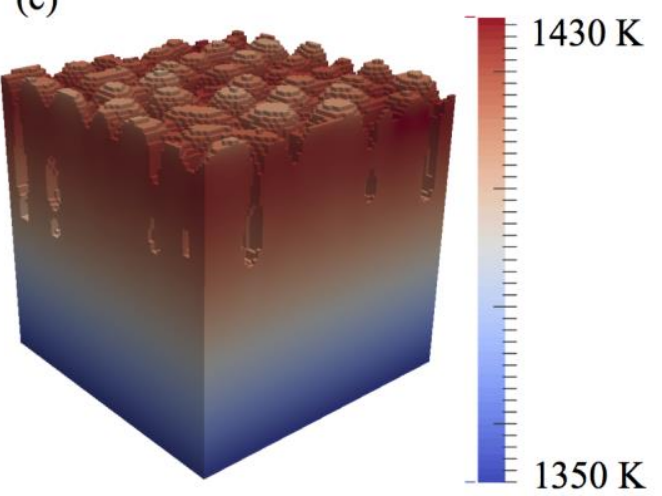

Figure 6. Simulated PVD on bicrystal substrate with $A_{y}=-0.6$ and $T=1350 \mathrm{~K}$ at time step $10^{7}$ where (a) is the phase-field, (b) is the underlying multiphase field, and (c) is the temperature field. All data is plotted for regions where $f \geq 0.5$. 
(a)

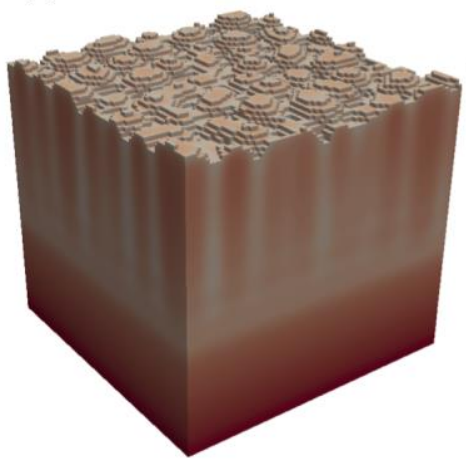

(b)

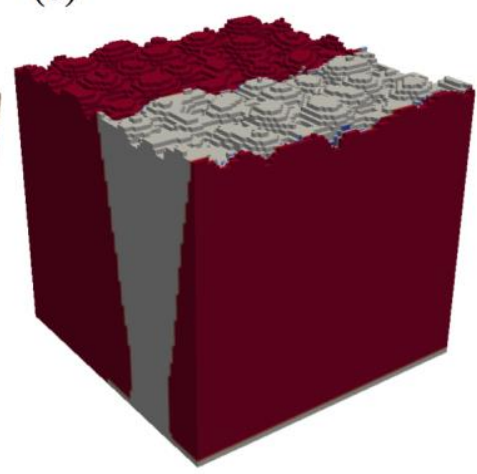

(c)

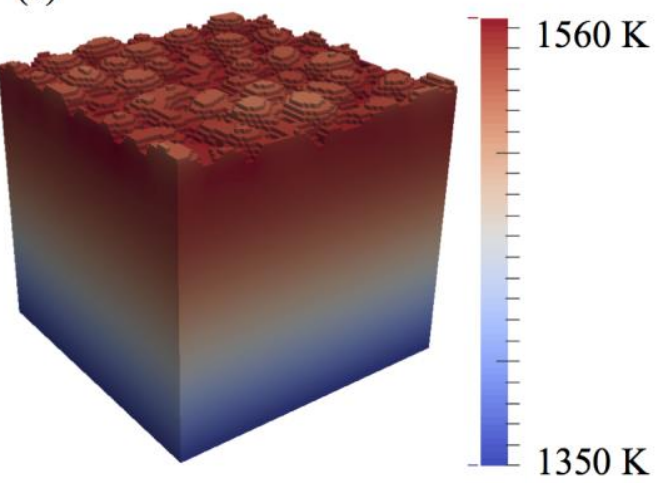

Figure 7. Simulated PVD on a bicrystal substrate with $A_{y}=-2.5$ and $T=1350 \mathrm{~K}$ at time step $5 \cdot 10^{6}$ where (a) is the phase-field, (b) is the underlying multiphase field, and (c) is the temperature field All data is plotted for regions where $f \geq 0.5$. 